\title{
Consensus Formation in Networked Groups
}

\author{
Carlo Martini*
}

April 1, 2010

\begin{abstract}
This paper applies the theory of networks to the problem of how agents should assign weights to other agents in the Lehrer-Wagner model for consensus formation. The LehrerWagner theory of consensus is introduced, and the problem of weight assignment is highlighted as one of the open problems for the theory. The paper argues that the application of the theory of networks to the Lehrer-Wagner model constitutes an interesting and fruitful option, among others, for the problem of weight assignment.
\end{abstract}

\section{Social epistemology and consensus models}

How consensus is formed, that is how situations of disagreement are resolved, is a central topic in social epistemology. Traditionally, epistemologists have been concerned, among other topics, with the problems related to belief formation from an individual perspective, but there is also a very important social dimension to that phenomenon.

The idea that group judgment has important epistemic advantages over individual judgment has been treated from numerous perspectives, although the formal treatment of the problem dates back to only a few centuries, with the marquis de Condorcet and the now renown theorem named after him, the Condorcet jury theorem (see List and Goodin 2001; Young 1988). Since then, research in social choice theory, psychology, and social epistemology has highlighted the epistemic advantages as well as disadvantages of group deliberation and group decision making (see Kitcher 1990; Aaken et al. 2004; Goldman 1999).

* Tilburg Center for Logic and Philosophy of Science, Tilburg University, PO Box 90153, 5000 LE, Tilburg (The Netherlands) - http://www.martinicarlo.net - c.martini@uvt.nl. 
It is in the context just outlined that in the past half century formal methods for consensus have been developed and studied. Consensus is particularly difficult to obtain with formal methods, as it involves not only the aggregation of individual beliefs or attitudes, but also the endorsement of such attitudes by all the members of a group. This is the main characteristic that distinguishes consensus models from voting procedures in general.

One of the most important works on consensual and its formal treatment is in a number of studies conducted in the late 70 s and early $80 \mathrm{~s}$ by the philosopher Keith Lehrer and the mathematician Carl Wagner - to cite a few, Wagner (1978), Lehrer (1976) and especially their joint Lehrer and Wagner (1981). Those works laid the foundation for what is now known as the Lehrer-Wagner model for consensus.

That model, together with the many discussions and issues that it raises, will be the central topic of the following sections.

\section{The Lehrer-Wagner Model}

Lehrer and Wagner (1981) develops a consensus model based on the convergence properties of Markov Chains. The model model produces consensus by means of iterated weighted averaging of the beliefs that a group's members may have on an issue object of disagreement. There are essentially three stages in the model, which will be illustrated in the following paragraphs.

In the first stage the agents in the model (the disagreeing agents, or parties) assign a certain measure $m_{i j}$ to themselves and to all others, where $m \in(0,1)$, and where $i$ is the agent assigning the measure, and $j$ is the agent receiving it. These measures form a $N \times N$ matrix $W$, with entries $w_{i j}$, where $N$ denotes the size of the group and each raw $W_{i *}$ is normalized, that is, $w_{i j}=\frac{m_{i j}}{\sum_{j=1}^{n} N_{i j}}$. The matrix $W$ is called the "matrix of weights" of the Lehrer-Wagner model.

$$
W=\left(\begin{array}{cccc}
w_{11} & w_{12} & \ldots & w_{1 N} \\
w_{21} & w_{22} & \ldots & w_{2 N} \\
\ldots & \ldots & \ldots & \ldots \\
w_{N 1} & w_{N 2} & \ldots & w_{N N}
\end{array}\right)
$$

In the second step, agents provide their judgment $p$ on the subject matter on which the group is deliberating. These judgments form a column of numbers (for instance, probabilities) $P$, with entries $p_{i}$, as exemplified below. 


$$
P=\left(\begin{array}{c}
p_{1} \\
p_{2} \\
\cdots \\
p_{N}
\end{array}\right)
$$

In the final stage, consensus is produced by multiplying the matrix of weights $k$ times, and then by the column of probabilities $P$ : that is, $W^{k} P$. A theorem shows that, under certain conditions, the values of the obtaining column $P_{C}$ will be equal to each other as the powers $k$ of $W$ rise.

$$
\text { (The Consensual probabilities column) } \quad P_{C}=W^{k} P \text { for } \quad k \rightarrow \infty
$$

The conditions for convergence ${ }^{1}$ of the values of $P$ are that the weights in each row of $W$ be normalized (see above), and that the matrix $W$ be not reducible ${ }^{2}$.

The reason why the model is more than a pooling algorithm for combining information cannot be easily summarized, and defending the model as a consensus model is beyond the scope of this paper. In short, the idea is that agents in the Lehrer-Wagner model are giving each other a weight, as an indication of their willingness that the group's opinion be a function of the opinions of all members in the group (Wagner 1978).

The difference from a simple weighted average is that Lehrer-Wagner works by making individuals converge slowly on a consensual value, rather than amalgamating all the individual information in a one-step procedure. However, what exactly the meaning of consensus is, in the Lehrer-Wagner framework, is a controversial matter and cannot be investigated in details in this paper ${ }^{3}$. In this paper, I assume that the model produces a real consensus, whatever the meaning of 'consensus' may be, and the rest of the discussion will focus on the problem of how agents in the model assign weights to each other.

\footnotetext{
${ }^{1}$ Proof of convergence under the stated conditions is omitted here, but can be found in Lehrer and Wagner (1981, pp. 129-133) and in Golub and Jackson (2007).

${ }^{2}$ More on the condition of reducibility will be said in section 3 , while discussing a possible method for assigning weights. For the mathematical notion of reducible matrices see Meyer (2000, pp. 209, 671).

${ }^{3}$ The interested reader can refer to Goodin (2001); Lehrer (2001); Bradley (2006) for some examples of the controversy.
} 


\section{The problem of weight assignment in the Lehrer-Wagner model}

How agents are to assign weights to one another remains one of the major unsolved problems in the Lehrer-Wagner model. Lehrer and Wagner (1981) does not give a strategy or algorithm for assigning weights, although four different examples of possible assignments are given. The first three examples involve some mechanical procedures for aggregating information and, in those, weights are not meant to represent trust or confidence in other agents' judgments, but rather some more or less objective quantity. The fourth example, on the other hand, makes weights dependent on subjective assignments.

When a decision problem involves neither highly structured estimation subject to a prior analysis of weighting schemes, as in the examples 1 and 2 above, nor a statistical record of past performance, as in the preceding example, then the choice of weights becomes a subjective enterprise. (Lehrer and Wagner 1981, 140)

The idea of a subjective assignment of weights seems to be what drives the consensual nature of the model (see Lehrer 1976), nevertheless making the assignment of weights a "subjective enterprise", rises a number of problems that have been pointed out in the subsequent literature on consensus.

One of the main critical stances on subjective assignment of weights is in Regan et al. (2006); the paper takes the Lehrer-Wagner model as a practical option for consensus seeking in medium-sized committees, such as the one involved in the case study the authors analyze. Their case study involves a panel of experts who are to formulate a list of criteria to be used in the selection of urban open spaces for a Californian environmental conservation project.

The problem is neither an entirely scientific one, because the list of criteria involves considerations too complex and multi-disciplinary for relying entirely on "hard science", nor entirely subjective, since important factors for evaluation need to be assessed on the basis of specific expert knowledge. Moreover, the agreement will most likely be influenced by stakeholders' interests and personal preferences.

In such context, it is argued in Regan et al. (2006), the Leher-Wagner model could provide a useful framework in order for the committee to achieve a consensus that takes into consideration not only the opinions of each expert, but also the weights associated with their opinions. As the authors 
note however, asking experts to subjectively assign weights to each other would be open to a number of theoretical and practical problems. In the first place, it would be a very impractical task to ask each member of the committee to assign a weight to all his or her fellows. Secondly members may hide their true agendas in order to manipulate the results from the model ${ }^{4}$. Thirdly, Regan et al. (2006) provides an argument that is reported in full below as it is difficult to summarize.

Third, and most important, the assignment of a numerical value on a person's degree of respect for each of the other members in the group is abstract and provocative. While most people would agree that they have different degrees of respect for, or agreement with, other group members' positions, translating that to a numerical value is non- trivial. Furthermore, group members may feel reluctant to explicitly quantify degrees of respect for other group members, or reveal their true weight of respect, as it could lead to rifts and ill feeling within the group. (Regan et al. 2006, 172)

For these reasons, the authors propose a method for assigning weights based on the relative distance of two agent's opinions. In other words, the weight $w_{i j}$ that agent $i$ assigns to agent $j$ will be a function of the distance between agent $i$ 's and agent $j$ 's opinions ${ }^{5}$.

A similar suggestion has also been put forth in Hegselman and Krause (2002). There, the authors present the Bounded Confidence model, a model for consensus similar to the Lehrer-Wagner ${ }^{6}$, and suggest that agents aggregate their opinion only with those other agents whose opinion is at a certain distance $\varepsilon$ from their own. In other words, the admitted weights are only 0 and 1 - agent $i$ will assign weight 1 to agent $j$ if and only if agent $j$ 's opinion lies within the "confidence interval" ' $\pm \varepsilon$ ' from hers, otherwise, she will give agent $j$ weight 0 .

Both the proposals in Regan et al. (2006) and Hegselman and Krause (2002) have a number of advantages, in particular, they solve the problems

\footnotetext{
${ }^{4}$ Nurmi shows that the Leher-Wagner model, when weights are assinged subjectively, is manipulable (see Nurmi 1985, 15: Proposition 1).

${ }^{5}$ Details on the function and derivation of weights are left to the interested reader (see Regan et al. 2006, 172).

${ }^{6}$ In fact, the Bounded Confidence model is a special case of the extended version of the Lehrer-Wagner, the presentation of which was omitted from section 2; the interested reader can refer to chapter 4: The extended model in Lehrer and Wagner (1981).
} 
highlighted in Regan et al. (2006). Weights are no longer assigned subjectively, thus avoiding, in part, manipulability of the assignment, and are derived from the information about the agents' opinions, thus providing an economic, easily quantifiable, and "sentiment free" measurement.

There are, however, also drawbacks to the that proposal. For example, imagine a case in which the decision on which the group is seeking agreement is, by nature, highly polarizing (e.g. people feel very highly about the outcome of the decision). The goal is to obtain a solution that be not only the win of a majority, but a function of all the group member's opinions.

Adopting either of the two aforementioned solutions would not achieve the goal of promoting agreement, rather the opposite effect of polarizing the two groups. In particular, an extreme type of manipulation is still possible with the Regan proposal: members of the two subgroups may conceal their true opinion and decide to give an extreme opinion ( 1 or 0$)$, knowing that it is opposite to the opinion of the other subgroup. If the other subgroup does the same, the matrix will "polarize" 7 into two submatrices which will converge independently to two different values ${ }^{8}$.

The Bounded Confidence model has similar problems. As Hegselman and Krause (2002) shows, in a important number of cases (if $\varepsilon$ is "large enough"), the model converges to two, or more, independent opinions, meaning that the original opinions of the group's members stabilize on multiple non-communicating convergent paths (see Hegselman and Krause 2002, 1020).

A possible reply to the challenges illustrated above is that it is pointless to seek for the ultimate method that solves all problems. In general, however, there seems to be a question to be asked about the rationality of deriving weights from distance in opinion, method that, in different ways, both Regan et al. (2006) and Hegselman and Krause (2002) adopt. The method is rational insofar as it is rational to expect that people will tend to converge towards those positions that are closer to theirs ${ }^{9}$. It is also a very economical

\footnotetext{
${ }^{7}$ In technical language, a matrix so contructed is said to be reducible. Reducible matrices do not converge to a unique value but to $n$ values, where $n$ is the number of submatrices that can be produced from the main matrix. For more details on the condition see Meyer (2000, pp. 209, 671).

${ }^{8}$ That this type of manipulation is possible can be seen from the function for the derivation of weights in Regan et al. (2006, 172): $w_{i j}=\frac{1-\left|p_{i}-p_{j}\right|}{\sum_{j=1}^{n} 1-\left|p_{i}-p_{j}\right|}$. If $i$ 's opinion is 1 and $j$ 's opinion is 0 , then $w_{i j}$ will be a null weight

${ }^{9} \mathrm{I}$ have so far taken both methods in Regan et al. (2006) and Hegselman and Krause (2002) to be normative in character. Whereas this is quite uncontroversial for the former, it is arguable whether the latter should be taken as a normative model. In principle, nonetheless, there seems to be no reason for prohibiting that it be taken normatively,
} 
method, as all the information that is needed for convergence is already present in the opinions of the group.

Nonetheless, in light of some of the problems illustrated below, it should be taken as one of a number of possible solutions, each of which has advantages as well as disadvantages. It is in the light of that that in the next section I will present an alternative method for assigning weights, one that seems particularly fruitful for counterbalancing, for example, the dynamics that tend to make a group split on a very polarizing issue.

\section{Deriving weights from a network's structure}

\subsection{Social influence and networks}

The method for the assignment of weights suggested in this section take its rationality from the observation that, in real life, groups do not come in the idealized form that is often assumed by most consensus models. Lehrer and Wagner (1981) assumes that agents give honest assignments of weights.

[...] if, on the contrary, the weights represent an egoistic attempt to manipulate social decisions making, then it is unacceptable to use those weights though they were a disinterested summary of information." (Lehrer and Wagner 1981, 74).

However, honest assignments cannot be taken as a realistic assumption. A member of a group may tend towards the opinion of other members not necessarily because those opinions are similar to hers, but also for a number of other possible reasons, e.g. political or economic interest, kinship, etc. In other words, in several concrete scenarios, people are not, so to speak, "on equal grounds", but rather "networked", to wit, organized in a structure (a network) in which the degree of connectedness of different agents varies depending on that agent's position in the network.

Network theory ${ }^{10}$ has recently drawn attention to the phenomenon of network formation and on how a network can affect the information flow in a group. This literature has evolved especially in economics and sociology; for example, DeMarzo et al. (2003) addresses the problem of how persuasion

regardless of the original authors' intentions. Besides, also from a descriptive point of view, weights need not be derived as a function of the distance of two agents' opinions - sometimes we give a high weight to people who are close to us (a friend or a business partner) even when their opinion is not similar to ours.

${ }^{10}$ For a recent comprehensive treatment of networks in economic and sociology see Jackson (2008). 
bias can be more effective depending on how well-connected an agent is in the group.

Biases, persuasion and the structure of a certain network can arise from disparate situations - a city council's members may be linked more or less strongly by political, economic and even family-related interests. Similarly, the members of an environmental panel may be linked by reasons of national interest, ideology or a number of other factors that will sway such opinions in one sense or another.

Golub and Jackson (2007) proposes a convergence model in which the flow of information in the group is conveyed through the existent network. They answer the question of which networks allow a deliberative group to reach consensus and necessary and sufficient conditions for convergence are provided. Golub and Jackson's approach however, does not allow agents to give different weights to one another if they are equidistant in the network.

The method suggested in this paper, on the other hand, allows for more flexibility, viz. weights are not fixed for all links but are: a) dependent on the total distance (in number of links) between agents and b) minimal variations are allowed to occur in the attribution of weights even when agents' mutual positions are equidistant.

\subsection{Network dependent weights}

The idea behind a derivation of weights from the underlying network structure of the deliberating group is that there are scenarios in which agents assign weights based on their preferences for, or biases towards, other agents.

Imagine the following scenario in which the group is composed of diplomats from different countries. For simplicity I will assume that each agent (each diplomat) has exactly two proximate neighbors. Agent $A$, is neighbor with agents $B$ and $C, B$ will then be neighbor with $A$ (the relation of being neighbor is symmetric) and $D, C$ on the other hand is neighbor with $A$ and $E$. Again, for simplicity, I assume that the group is small and that it is closed, that is, there is a member $F$ that has $E$ and $D$ as neighbors. 
Figure 1: a six-node network

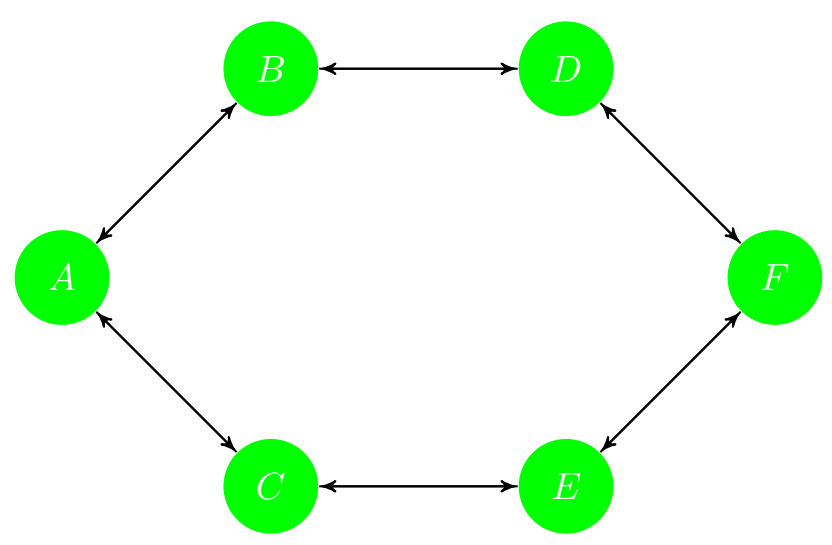

Neighboring countries, in some cases, have common interests: trade, military security, environmental safety, etc. As the distance between two countries increases, however, those factors might play less and less a role in their preferences towards one another.

From figure 1, a list of instructions for the derivation of weights can be formulated, as illustrated in table 1.

Table 1: Weight-derivation for a six node graph

1. $w_{y x} \in(a, b) \Longleftrightarrow \#$ edges between $x$ and $y$ is 0 (case for $x=y$ )

2. $w_{y x} \in(b, c) \Longleftrightarrow \#$ edges between $x$ and $y$ is 1 (where $a \gtrless b$ )

3. $w_{y x} \in(c, d) \Longleftrightarrow$ \# edges between $x$ and $y$ is 2 (where $b \gtrless c$ )

4. $w_{y x} \in(d, e) \Longleftrightarrow$ \# edges between $x$ and $y$ is 3 (where $c \gtrless d$ )

Table 1 gives a simple set of rules for the derivation of weights based on the relative distance (in number of nodes) from agent to agent. For example, if $a=1 ; b=0.8 ; c=0.5 ; d=0.3 ; e=0$, then $w_{a d} \in(0.5,0.3)$ because agent $A$ is two links away from agent $D$. According to the schema, an agent will give herself a higher weight, her immediate neighbor a slightly lower weight, her next neighbor an even lower weight, and so on. The schema can be reformulated for any number of nodes in a ring-shaped network. 


\section{$5 \quad$ Examples}

\subsection{A balanced network}

Hartmann et al. (2009) analyzes the case of a ring-shaped network, like the one presented in section 4.2 , in order to provide a formal definition of epistemic peers and investigate the dynamics of consensus formation. Initially, the paper assumes a stricter schema for the assignment of weight, such that if agent $A$ is distant $x$ nodes from agent $B$, then there is only one possible weight that $w_{a b}$ and $w_{b a}$ can take. In other words, with reference to table 1 , each " $\ldots \in(a, b) \ldots$ " [or $(b, c)$, etc.] is substituted by “ $\ldots=\alpha$ $\ldots "$ [or $\beta$, etc.], where $\alpha, \beta$, etc. are fixed values between 0 and 1 .

It is possible to show (see Hartmann et al. 2009, 116: Theorem 1) that the consensual results deriving from the model are equal to the arithmetic average of the values in the column $P$ (see above, section 2). In the same paper, it was shown that also relaxing the schema for the assignment of weights (as from Table 1, above), the results are robust, that is, the consensual values will be approximately equal $(\approx)$ to the arithmetic average of the column $P$.

What those result mean is that whenever the group forms a symmetric network, like the one of figure 1 (or any isomorphic extension of that network with more agents), the different weights assigned by agents to other agents balance each other out and the situation is equivalent to that of a group where agents assign no weights to one another.

In Hartmann et al. (2009), the analysis of the ring-shaped network in combination with the Lehrer-Wagner model was used in order to justify an "equal weight view" among epistemic peers (see Kelly 2005; Elga 2007). In the context of this paper, however, the idea is to provide a rationale for the derivation of weights from the underlying network structure, both from a normative and a descriptive point of view.

More on the normative and descriptive implications of the analysis of this paper will be said in section 5.3.

\subsection{Other networks}

The example provided in section 5.1 is only one of numerous possible network formations that can in principle be studied. A thorough analysis of the dynamics of consensus formation with the Lehrer-Wagner model has yet to be carried out and is beyond the scope of this paper. The following remarks will serve as an illustrations of the many theoretical possibilities that such analysis could disclose. 
The ring-shaped network is an example of an extremely regular network, in which all the weights balance out. It could, as said before, represent a number of countries that have a bias towards their neighbors, but whose global effect would not influence the results of a consensus.

Other networks, however, are also of theoretical interest. For instance, there may be groups in which one of the agents plays the role of the leader, and to whom all other agents assign a high degree of respect or confidence (a high weight). An example of this is what Elga calls 'gurus', that is "people to whom we should defer entirely" (Elga 2007, 478). Identifying the presence of a guru in a group might have important epistemic implications.

The epistemic value of the presence of a guru was one of the points left open in Hartmann et al. (2009): "[...] we did not address the question of whether the leader bias is beneficial or not - this depends on the leader's factual competence and honesty. The Lehrer-Wagner model is silent on these questions." (Hartmann et al. 2009, 120).

The Lehrer-Wagner model, when weights are assigned subjectively, is not sensitive to the presence of a leader in the group. However, in a star-like network (see Figure 2), the consensus will be biased towards the opinion of the leader ${ }^{11}$.

Figure 2: a nine-node ring-shaped network

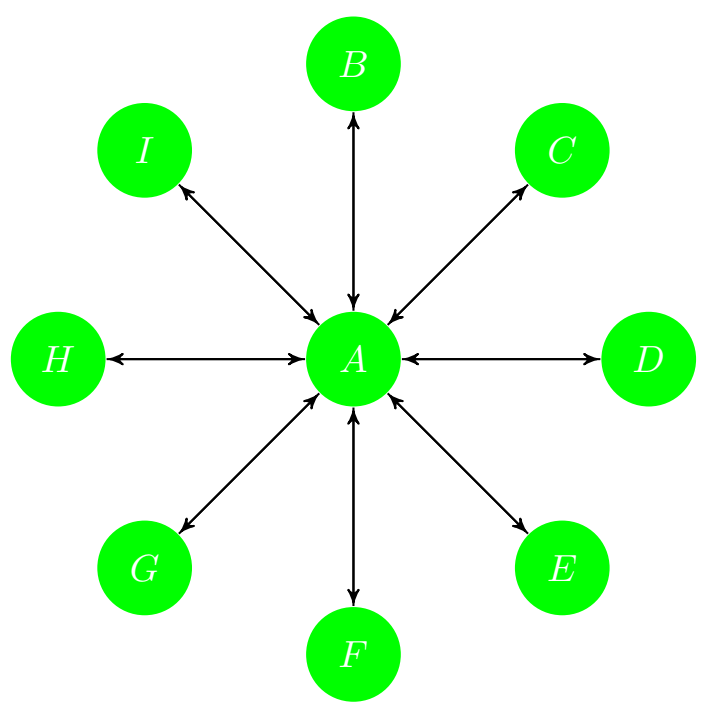

Deriving weights from a given star-shaped network would, on one hand, allow us to represent a real case of consensus formation where a leader should

\footnotetext{
${ }^{11}$ For an explanation of this see Hartmann et al. (2009, 120: Theorem 3)
} 
be present. On the other hand, from a normative point of view, it is to be noted that the presence of the leader could be a positive one or a negative one, depending on whether we deem the opinion of the leader in question a "good" opinion or not.

With the scheme for deriving weights exemplified in table 1, if properly modified, it is possible to balance the weights that agents assign to one another so that the opinion of the leader will be weighted higher or lower, depending on which of the two we deem as the appropriate strategy. For example, if we deem the leader to be a negative influence on the group, the schema for the assignment of weights could be built along the lines of table 2 below.

Table 2: Weight-derivation for a nine node graph

1. $w_{y x} \in(a, b) \Longleftrightarrow \#$ edges between $x$ and $y$ is 0 (case for $x=y$ )

2. $w_{y x} \in(b, c) \Longleftrightarrow \#$ edges between $x$ and $y$ is 2 (where $a \gtrless b$ )

3. $w_{y x} \in(d, e) \Longleftrightarrow \#$ edges between $x$ and $y$ is 1 (where $d \ll c$ )

The derivation in table 2 implies that the influence of a leader, that would normally sway the results of the consensus, is diminished. Agents will still be giving more confidence (or preference) to the leader, due to the preference structure of the network, but their opinions towards the leader, will be automatically scaled down. The idea is that the consensus should be a measure of an agent's independent opinion on the matter under consideration, so that factors affecting that independence, if possible to detect, should be minimized.

\subsection{Epistemic implications of network-dependent weights}

At the end of section 3 it was said that the method for deriving weights suggested in this paper should be taken as one of many possible methods, each of which will have advantages as well as drawbacks. In this section I will enumerate a number of reasons for justifying weight derivation on the basis of the underlying network structure, both from a normative and descriptive take on the model.

I start from the normative point of view, which was the one tacitly assumed in section 3 (see footnote 9 ). The normative interpretation of the Lehrer-Wagner model is the one endorsed in Regan et al. (2006). According to that paper, weights should be assigned as a function of the distance between agents' opinions. This is rational in those cases in which agents assign 
higher weights to agents with opinions similar to their own, for example, we may think that musicians may assign higher value to other musicians with similar tastes.

That scenario, however, need not always be the case. As I justified in section 4.2 we can imagine cases in which we give a higher weight to someone who is "close" to us, or whom we view as the leader, no matter what her opinion on the matter under deliberation is. In all these cases the problem is whether such preference, the influence of the network on the group's decision, is epistemically advantageous or not.

The main reason for normatively adopting a network-based derivation of weights is that it is in principle possible to exploit the epistemic advantages of a certain network or minimize its influence, depending on which one is the recognized situation.

Some interpretations, however, see the Lehrer-Wagner model as a descriptive model of consensus formation ${ }^{12}$. In the second part of this section I will justify a network-depended assignment of weights from a descriptive point of view.

A descriptive interpretation of the Lehrer-Wagner model claims that the model is a representation, however idealized, of how consensus is formed, rather than a deliberating method that groups should use in order to achieve agreement.

Agents often seek consensus by deliberating and putting their opinions together, and trying to come up with a "group opinion". But the process is not one of pure amalgamation, as some pooling algorithms may imply, rather it is, normally, an iterative process, in which agents come closer and closer to each other's opinions until a single one emerges as the consensual one. Moreover, it is reasonable to assume that agents will tend to assign (perhaps unconsciously) some trust or confidence to other agents. The higher the degree of trust I assign to agent $i$, the more $i$ 's opinion will influence my own opinion while moving towards the sought consensus.

How the mathematics of the Lehrer-Wagner model applies to the situation just depicted is fairly straightforward. The iterative nature of the deliberating process is captured by the subsequent rounds of the model towards convergence, and the measure of trust is represented by the weights that agents assign to each other. For mathematical reasons, at each round my opinion will be driven mostly by the opinion of those agents to whom I

\footnotetext{
${ }^{12}$ Indeed early versions of consensus models that use the properties of convergent Markov chains make reference to DeGroot (1974), who takes the model to be descriptive in character.
} 
have give higher weights - this is the idea that my opinion at each round will be affected mostly by those agents that I deem more trustworthy.

An interesting extension of the idea of describing consensus formation through the Lehrer-Wagner models is to apply the theory of networks to its system of weight assignment. Some networks will, in concrete cases, affect the formation of consensus, and it is in principle possible to study the effects of the network on the consensus that is produced.

A very straightforward example will clarify the latter point. Figure 3, represents a group in which two subgroups are present, groups $(B, D, E, F)$ and $(C, G, H, I)$, and the two are linked by a "mediator" $(A)$.

Figure 3: a double ring network with mediator

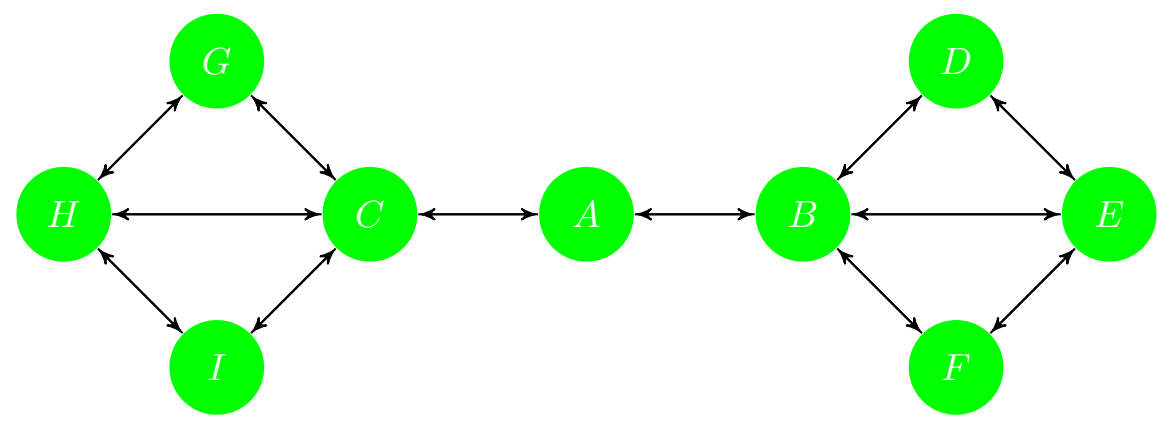

In this case it is clear the mediator plays a central role in the formation of a consensus, as it is the only one that shares a certain measure of trust or confidence with the two subgroups. Indeed, were $A$ to be deleted from figure 3 , together with the two edges that link it with $B$ and $C$, then the two subgroups would not converge to a consensus, but to two independent opinions, one driven by the communication of trust in subgroup $(B, D, E, F)$, and the other driven by communication of trust in subgroup $(C, G, H, I)$.

\section{Conclusions}

This paper started from considering a well known model for consensus formation, the Lehrer-Wagner model. The use of a formal model for the analysis of consensus formation, as well as the development of a normative theory of consensus, is a very fruitful approach for the study of consensus in social epistemology.

The model developed by Lehrer and Wagner, however, besides providing such useful tool of investigation, also opens a number of problems, among 
which the problem of how a group of agents should assign weights to one another, or how to represent the distribution of weights (trust, confidence) that is reasonable to assume takes place in a group seeking consensus.

The paper does not claim that there is a one-fit-all solution for determining weights - for example, the methods suggested in Regan et al. (2006) and Hegselman and Krause (2002) seem to be fit for certain situations but unfit for others.

Using some ideas from the theory of networks for determining weight assignment among agents, however, seems to be a fruitful approach for those of cases in which we wish to represent consensus in "networked groups". The examples provided in this paper, as well as the analysis in Hartmann et al. (2009), constitute only a small fraction of a potentially large number of possible applications, and further lines of investigation, in the topic of consensus formation by means of formal models like the Lehrer-Wagner.

\section{References}

Aaken, Anne van, Christian List and Christoph Luetge (eds.) (2004) Deliberation and Decision: Economics, Constitutional Theory and Deliberative Democracy. London: Ashgate.

Bradley Richard (2006) 'Taking Advantage of Difference of Opinion' Episteme: A Journal of Social Epistemology 3 (3): 141-155.

DeGroot, M. H. (1974) 'Reaching a consensus' Journal of the American Statistical Association 69(345): 118-121.

DeMarzo, Peter M., Dimistri Vayanos and Jeffrey Zwiebel (2003) 'Persuasion Bias, Social Influence and Unidimensional Opinions' The Quarterly Journal of Economics (August): 909-968.

Elga, Adam (2007) 'Reflection and Disagreement' Noûs 41: 478-502.

French, J. R. J. (1956) 'A formal theory of social power' Psychological Review 63(3): 181-194.

Goldman, Alvin (1999) Knowledge in a Social World. Oxford: Oxford University Press.

Golub, Benjamin and Matthew O. Jackson (2007) 'Naïve Learning in Social Networks: Convergence, Influence, and the Wisdom of Crowds' Working Papers Series FEEM Working Paper No. 64.

Goodin, Robert E. (2001) 'Consensus Interruptus' The Journal of Ethics 5: 121131. 
Hartmann, S., C. Martini, and J. Sprenger (2009) 'Consensual decision-making among epistemic peers' Episteme 6(2): 110-129.

Hegselmann, R. and U. Krause (2002) 'Opinion Dynamics and Bounded Confidence: Models, Analysis and Simulation.' Journal of Artificial Societies and Social Simulation 5(3).

Hegselmann, R. and U. Krause (2005) 'Opinion Dynamics Driven by Various Ways of Averaging' Computational Economics 25: 381-405.

Hegselmann, R. and U. Krause (2006) 'Truth and Cognitive Division of Labour: First Steps towards a Computer Aided Social Epistemology' Journal of Artificial Societies and Social Simulation 9(3).

Jackson, Matthew O. (2008) Social and Economic Networks. Princeton: Princeton University Press.

Kelly, Thomas (2005) 'The Epistemic Significance of Disagreement' In: J. Hawthorne and T. Szabo (eds.) Oxford Studies in Epistemology 1, pp. 167-196. Oxford: Oxford University Press.

Kitcher, Philip (1990) 'The Division of Cognitive Labor' The Journal of Philosophy, 87(1): 5-22.

Lehrer, Keith and Carl Wagner (1981) Rational Consensus in Science and Society. Dordrecht: Reidel.

Lehrer, Keith (1976) 'When Rational Disagreement is Impossible' Noûs 10 (3): 327-332.

Lehrer, Keith (2001) 'The rationality of dissensus' The Journal of Ethics 5: 133137.

List, C. and R. E. Goodin (2001) 'Epistemic Democracy: Generalizing the Condorcet Jury Theorem', Journal of Political Philosophy 9: 277-306.

Meyer, Carl D. (2000) Matrix Analysis and Applied Linear Algebra, Philadelphia: Society for Industrial and Applied Mathematics (SIAM).

Nurmi, Hannu (1985) 'Some properties of the Lehrer-Wagner Method for Reaching Rational Consensus' Synthese 62: 13-24.

Regan, Helen M., Mark Colyvan, Lisa Markovchick-Nicholls (2006) 'A Formal Model for Consensus and Negotiation in Environmental Management' Journal of Environmental Management 80: 167-176.

Wagner, Carl (1978) 'Consensus through respect: a model of rational group decision-making' Philosophical Studies 34: 335-349.

Young, H. P. (1988) 'CondorcetÕs theory of voting', American Political Science Review 82: 1231-1244. 